\title{
Systematic Review and Proportional Meta-Analysis of Endarterectomy and Endovascular Therapy with Routine or Selective Stenting for Common Femoral Artery Atherosclerotic Disease
}

\author{
Khalid Hamid Changal $\left(1 D,{ }^{1}\right.$ Mubbasher Ameer Syed, ${ }^{2}$ Tawseef Dar, ${ }^{3}$ \\ Muhammad Asif Mangi, ${ }^{2}$ and Mujeeb Abdul Sheikh (iD ${ }^{4}$ \\ ${ }^{1}$ Internal Medicine, Mercy Health St. Vincent Medical Center, Toledo, OH, USA \\ ${ }^{2}$ Cardiovascular Medicine, University of Toledo College of Medicine and Life Sciences, Toledo, OH, USA \\ ${ }^{3}$ Cardiology Division, Massachusetts General Hospital, Harvard Medical School, Boston, Massachusetts, USA \\ ${ }^{4}$ Cardiovascular Medicine and Interventional Cardiology, University of Toledo College of Medicine and Life Sciences, \\ 3065 Arlington Ave. Toledo, $\mathrm{OH} 43614$, USA
}

Correspondence should be addressed to Mujeeb Abdul Sheikh; Mujeeb.Sheikh@utoledo.edu

Received 6 December 2018; Revised 4 March 2019; Accepted 28 March 2019; Published 14 April 2019

Academic Editor: Yuichiro Maekawa

Copyright (c) 2019 Khalid Hamid Changal et al. This is an open access article distributed under the Creative Commons Attribution License, which permits unrestricted use, distribution, and reproduction in any medium, provided the original work is properly cited.

\begin{abstract}
Introduction. Common femoral endarterectomy (CFE) has been the therapy of choice for common femoral artery atherosclerotic disease (CFA-ASD). In the past, there was inhibition to treat CFA-ASD endovascularly with stents due to fear of stent fracture and compromise of future vascular access site. However, recent advances and new evidence suggest that CFA may no longer be a 'stentforbidden zone. In the light of new evidence, we conducted a meta-analysis to determine the use of endovascular treatment for CFAASD and compare it with common femoral endarterectomy in the present era. Methods. Using certain MeSH terms we searched multiple databases for studies done on endovascular and surgical treatment of CFA-ASD in the last two decades. Inclusion criteria were randomized control trials, observational, prospective, or retrospective studies evaluating an endovascular treatment or CFE for CFA-ASD. For comparison, studies were grouped based on the treatment strategy used for CFA-ASD: endovascular treatment with selective stenting (EVT-SS), endovascular treatment with routine stenting (EVT-RS), or common femoral endarterectomy (CFE). Primary patency (PP), target lesion revascularization (TLR), and complications were the outcomes studied. We did proportional meta-analysis using a random-effect model due to heterogeneity among the included studies. If confidence intervals of two results do not overlap, then statistical significance is determined. Results. Twenty-eight studies met inclusion criteria (7 for EVT-RS, 8 for EVT-SS, and 13 for CFE). Total limbs involved were 2914 (306 in EVT-RS, 678 in EVT-SS, and 1930 in CFE). The pooled PP at 1 year was $84 \%$ (95\% CI 75-92\%) for EVT-RS, 78\% (95\% CI 69-85\%) for EVT-SS, and 93\% (95\% CI 90-96\%) for CFE. PP at maximum follow-up in EVT-RS was 83.7\% (95\% CI 74-91\%) and in CFE group was $88.3 \%$ (95\% CI 81-94\%). The pooled target lesion revascularization (TLR) rate at one year was $8 \%$ (95\% CI 4-13\%) for EVT-RS, $19 \%$ (95\% CI 14-23\%) for EVT-SS, and 4.5\% (95\% CI 1-9\%) for CFE. The pooled rate of local complications for EVT-RS was 5\% (95\% CI 2-10\%), for EVT-SS was 7\% (95\% CI 3 to $12 \%$ ), and CFE was $22 \%$ (95\% CI 14-32\%). Mortality at maximum follow-up in CFE group was $23.1 \%$ (95\% CI 14-33\%) and EVT-RS was 5.3\% (95\% CI 1-11\%). Conclusion. EVT-RS has comparable one-year PP and TLR as CFE. CFE showed an advantage over EVT-SS for one-year PP. The complication rate is lower in EVT RS and EVT SS compared to CFE. At maximum follow-up, CFE and EVT-RS have similar PP but CFE has a higher mortality. These findings support EVT-RS as a management alternative for CFA-ASD.
\end{abstract}

\section{Introduction}

Atherosclerotic steno-occlusive disease of common femoral artery (CFA) in isolation is rare but often leads to symptomatic peripheral arterial disease [1]. In most instances, the atherosclerotic disease either extends beyond the CFA into contiguous arterial segments or is associated with multilevel disease proximally or distally. Historically, 
atherosclerotic steno-occlusive disease involving CFA is treated surgically with common femoral endarterectomy (CFE) which has so far remained the procedure of choice [2].

In the past, there was inhibition to treat common femoral artery atherosclerotic steno-occlusive disease (CFA-ASD) endovascularly with stents due to fear of stent fracture and compromise of future vascular access site. With significant advancements in the endovascular techniques, stent design, and adjunctive technologies, successful endovascular treatment of CFA-ASD is reported to be no longer considered a "stent forbidden zone" [2-7]. EVT of CFA disease has been shown to be associated with lower morbidity and mortality, shorter inpatient stay, improved patency, and faster recovery and in most cases requires local anesthesia only [2-7]. Paradigm shift to the treatment of CFA-ASD is limited by inconsistent results from earlier studies, mostly observational, examining the technical success and safety of EVT for treatment of CFA disease [2, 8, 9]. An older trial using bioabsorbable stents (BAS) showed worse oneyear primary patency and increased redo procedures in the stent group compared to CFE [10]. Newer studies that are using more advanced endovascular techniques and stents are showing comparable clinical outcomes with EVT and CFE [11]. Thus, the role of EVT in CFA-ASD is still a subject of debate [3-8]. We henceforth conducted a meta-analysis to compare endovascular treatment for CFA-ASD with common femoral endarterectomy.

\section{Materials and Methods}

We conducted a thorough search of Pubmed, Medline, Cochrane, Embase, and clinicaltrials.gov to identify studies evaluating different treatment approaches of CFA ASD. The review was done per the Preferred Reporting Items for Systematic Reviews and Meta-Analyses (PRISMA) guidelines. A comprehensive search strategy, using an exhaustive list of MeSH terms, words, and synonyms, was carried out to identify the relevant studies on therapeutic approaches (endovascular and surgical) for CFA-ASD (Appendix 1, supplementary file). The bibliographies of relevant review articles and eligible studies were examined to complete a comprehensive search of all the published studies.

Inclusion criteria were randomized control trials, observational studies, and prospective or retrospective studies evaluating an endovascular treatment or CFE for CFAASD. Studies that measured outcomes including primary patency (PP) and/or target lesion revascularization (TLR) were included. Animal studies, studies with incomplete data, studies published before the year 2000, case reports, case series with less than 10 patients, and studies published in languages other than English were excluded from the analysis. In view of lack of an adequate number of RCTs, we performed a pooled proportional meta-analysis of outcomes for different therapeutic approaches from all the included studies on CFA-ASD.

We divided the patients in the studies into three groups based on the treatment strategy used for CFA-ASD: endovascular treatment with selective stenting (EVT-SS), endovascular treatment with routine stenting (EVT-RS), and common femoral endarterectomy [CFE] (Figure 1). In EVTSS group primary treatment strategy was angioplasty only and stents were used only selectively. In EVT-RS group stenting with angioplasty was the primary treatment strategy. In CFE group endarterectomy of the common femoral artery was the primary treatment strategy and selected patients got additional endovascular treatment proximal or distal to CFA. CFE involves surgically opening the CFA longitudinally and peeling of the atherosclerotic plaque and in most cases involves patch angioplasty to close the vessel. PP, TLR, complications, and mortality were the main outcomes analyzed.

2.1. Data Collection. Two independent reviewers did the data collection [KC \& SM]. Conflicts were sorted out by discussion and adjudication by a third reviewer [TD]. We did an analysis of search results for inclusion and exclusion criteria before data extraction. Multiple key variables were extracted including but not limited to author, year of publication, country where study was done, design of study, total duration of study, demographics and comorbidities, clinical presentation (claudication vs. critical limb ischemia), anatomy of lesions, types of stents used, length of follow-up, patency at followup, and complications. If we found duplicate studies, the most complete and latest article reporting relevant outcomes of interest was included. When articles did not report the number of limbs involved, we assumed them to be the same as the number of patients in the study. For the two randomized controlled trials included in the study, data about CFE and stenting was extracted separately into respective groups.

2.2. Statistical Analysis. Primary patency and complication rates were treated as dichotomous variables with, respectively, 95\% confidence interval [CI]. Proportional meta-analysis was done using a random-effects model due to variations among the included studies and numerous uncontrolled variables. Forest plots were used to summarize the results. Each horizontal line in the forest plot represents a study. The length of this horizontal line represents 95\% CI of the corresponding study's effect estimate. The effect estimate is marked by a solid black square on the horizontal line, the size of which corresponds to the weight a study exerts in the meta-analysis. The overall pooled estimate is represented by the diamond shape at the bottom of each forest plot. A horizontal line through the diamond stands for the CI of pooled results. The statistical significance between different interventions was defined if their corresponding 95\% CI did not overlap. $\mathrm{I}^{2}$ statistic was used to assess statistical heterogeneity with an $\mathrm{I}^{2}>50 \%$ regarded as statistically significant. A p $<0.05$ was considered statistically significant for calculation of heterogeneity. This afforded a better measure of the consistency between the studies [35]. To assess publication bias we used Funnel plots performed by Egger tests. Asymmetric funnel plots are suggestive of publication bias. 


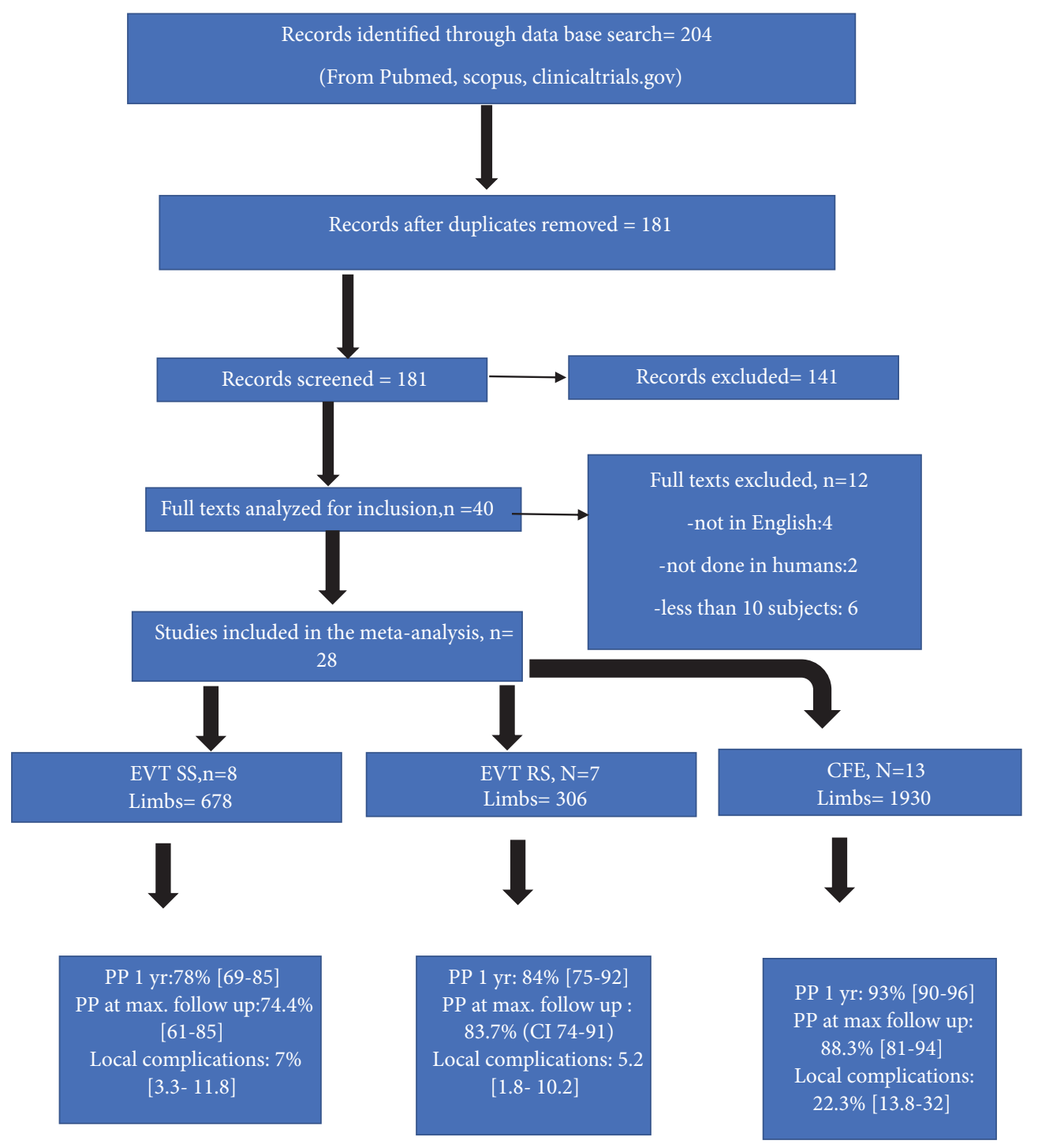

FIGURE 1: Flowsheet summarizing the selection of studies and main results.

\section{Results}

3.1. Study and Sample Characteristics. The search was performed from January 2000 to October 2017. After screening titles, abstracts, and excluding duplicate studies we obtained full-text copies of 167 studies relevant to EVT-SS, EVT-RS, or CFE for CFA-ASD. A total of 28 studies met all inclusion criteria (7 for EVT-RS, 8 for EVT-SS and 13 for CFE). Two RCTs had data on EVT-RS vs CFE and these were included to extract data into EVT-RS and CFE groups. Total of 2684 patients were included in the study (289 in EVT-RS, 646 in EVT-SS, and 1749 in CFE). The total number of limbs involved was 2914 (306 in EVT-RS, 678 in EVT-SS, and 1930 in CFE) [Figure 1].

3.2. Demographics and Comorbidities. Baseline demographics and comorbidities for the three study groups are given in Table 1 . Men formed the majority in all study groups $(61.7 \%$ in EVT-SS, 68.8\% EVT-RS, and 69.9\% in CFE). Hypertension was the most common comorbidity in all the study groups (84.9\% in EVT-SS, 83.3\% EVT-RS, and 85.6\% in CFE). Most of the comorbidities and risk factors were distributed evenly amongst the three groups with a few exceptions. Coronary artery disease was less prevalent in the EVT-RS group (55.2\% in EVT-SS, 36.3\% EVT-RS, and 60.8\% in CFE) whereas the EVT-SS group had fewer smokers (45.3\% in EVT-SS, 59.1\% EVT-RS, and $61.1 \%$ in CFE) but more patients with ESRD (27.5\% in EVT-SS, 17.4\% EVT-RS, and $11.8 \%$ in CFE).

3.3. Clinical Presentation. Clinical presentation was classified by Fontaine Classification [36] in some publications and by Rutherford Classification [37] in others. After reviewing data of all the included patients in the three treatment groups, we reclassified them either as Claudication [Fontaine Stagse I \& II | Rutherford Categories 0, 1, 2, and 3] or CLI [Fontaine Stages III and IV | Rutherford Categories 4, 5, and 6] patients. 
TABLE 1: Baseline demographics and comorbidities of patients in the different treatment groups.

\begin{tabular}{lccc}
\hline Variable & $\begin{array}{c}\text { EVT - Selective Stenting } \\
\mathrm{n}(\%)\end{array}$ & $\begin{array}{c}\text { EVT- Routine Stenting } \\
\mathrm{n}(\%)\end{array}$ & $\mathrm{n}(\%)$ \\
\hline Total studies & 8 & 646 & 14 \\
\hline Number of patients & 678 & 306 & 1756 \\
\hline Number of limbs & 68.4 & 68.8 & 1930 \\
\hline Mean Age [years] & $399(61.7)$ & $202(69.8)$ & $1299(73.9)$ \\
\hline Males & $247(38.3)$ & $87(30.2)$ & $457(26.1)$ \\
\hline Females & $253(39.1)$ & $102(35.2)$ & $736(41.9)$ \\
\hline Diabetes Mellitus & $357(55.2)$ & $105(36.3)$ & $1069(60.8)$ \\
\hline Coronary Artery Disease & $451 / 531^{\#}(84.9)$ & $241(83.3)$ & $1504(85.6)$ \\
\hline Hypertension & $317 / 531^{\#}(59.6)$ & $183(63.3)$ & $1106 / 1666^{*}(66.3)$ \\
\hline Hyperlipidemia & $241 / 531^{\#}(45.3)$ & $171(59.1)$ & $1074(61.1)$ \\
\hline Smokers & $84 / 305^{\circledR}(27.5)$ & $19 / 109(17.4)^{9}$ & $164 / 1379^{\wedge}(11.8)$ \\
\hline End Stage Renal Disease & $419(64.8)$ & $191(66.1)$ & $943(53.7)$ \\
\hline Claudication & $227(35.1)$ & $97(33.5)$ & $1021(58.1)$ \\
\hline CLI & &
\end{tabular}

[@ 3 out of 8 studies $\mid \# 7$ out of 8 studies $\mid * 12$ out of 13 studies $\mid \wedge 9$ out of 13 studies].

TABLE 2: Classification of lesions included in the different treatment groups.

\begin{tabular}{lccc}
\hline Variable & EVT - Selective Stenting & EVT- Routine Stenting & CFE \\
& $\mathrm{n}(\%)$ & $\mathrm{n}(\%)$ & $63(20.5)$ \\
\hline Type I Lesion [Inflow + CFA] & $84 / 574^{\#}(14.6)$ & $93(30.3)$ & $318 / 844^{\$}(37.6)$ \\
\hline Type II Lesion [Isolated CFA] & $310 / 652^{\#}(47.5)$ & $125(40.8)$ & $716 / 1668^{\Omega}(42.9)$ \\
\hline Type III Lesion [Outflow + CFA] & $265 / 574^{\#}(46.1)$ & $25(8.2)$ & $410 / 955^{\Omega}(42.9)$ \\
\hline Type IV Lesion [Bypass grafts and other lesions] & $26(3.8)$ & 0 \\
\hline
\end{tabular}

[\# 7 out of 8 studies $\mid \$ 10$ out of 13 studies | \& 12 out of 13 studies $\left.\right|^{\Omega} 11$ out of 13 studies].

The distribution of patients presenting with claudication versus CLI was $64.8 \%$ vs $35.1 \%$ in EVT-SS, $66.1 \%$ vs $33.5 \%$ in EVT-RS, and $53.7 \%$ vs $58.1 \%$ in CFE, which revealed that the patients in CFE group could have had more advanced disease in comparison to EVT groups (Table 1).

3.4. Lesion Characteristics. In the published studies various classification systems were used for describing lesion characteristics. We reviewed two classification systems described previously for CFA-ASD. Bonvini et al. [38] described CFA lesions based on Medina Classification that is used for bifurcation coronary artery disease. The second classification system described by Azema et al. [12] is based on inflow and outflow lesions and classifies CFA-ASD into 4 categories. We chose the latter for reclassifying the lesions in various treatment groups as it was more convenient to apply and easier to correlate with outcomes. Table 2 details the lesion types included in each treatment group. Isolated CFA lesions categorized as type II lesions were seen in $47.5 \%, 30.3 \%$, and $42.9 \%$ in EVT-SS, EVT-RS, and CFE groups, respectively.

3.5. Procedural Data. Total of 330 CFA stents were placed in EVT-RS group and majority were self-expanding stents (71.8\%). In EVT-SS group 123 CFA stents were placed, with $86.4 \%$ stents being self- expanding. A total of 552 stents were placed in CFE group during surgery, all of which were either proximal or distal to CFA. In EVT-RS group 24 stents were repunctured without any complication. Two stents were repunctured in EVT-SS group. In the endovascular group most procedures were performed by gaining the access in a contralateral cross-over fashion $(70.6 \%$ in EVT-SS and $78.1 \%$ in EVT-RS). Ipsilateral retrograde approach was used in $15.3 \%$ in EVT-SS and $12.4 \%$ in EVT-RS. In the CFE group, 48.9\% (944) procedures were CFE only, while 41.1\% (794) were hybrid procedures involving an additional endovascular procedure. Patch angioplasty was used in $72.1 \%$ (13 out of 14) whereas $5.6 \%(n=110)$ patients underwent concomitant IFBP (ilio-femoral-bypass-grafting). The procedural details for the three groups are illustrated in Table 3 .

3.6. Clinical Efficacy. We used primary patency and target vessel revascularization rates (as defined in Appendix 2) to be the measures of clinical efficacy for the different treatment strategies. The pooled proportion for primary patency (PP) at 1 year was $78 \%$ (95\% CI 69-85\%) for EVT-SS group, $84 \%$ (95\% CI $75-92 \%)$ for EVT-RS group, and 93\% (95\% CI 90-96\%) for CFE group (Figures 2-7). The CIs of EVTRS and CFE groups overlap and thus statistically significant difference could not be proven between these two treatment strategies. On the other hand, CFE showed clear advantage over selective stenting strategy [EVT-SS] in terms of PP at 1 year. The pooled target lesion revascularization (TLR) rates 


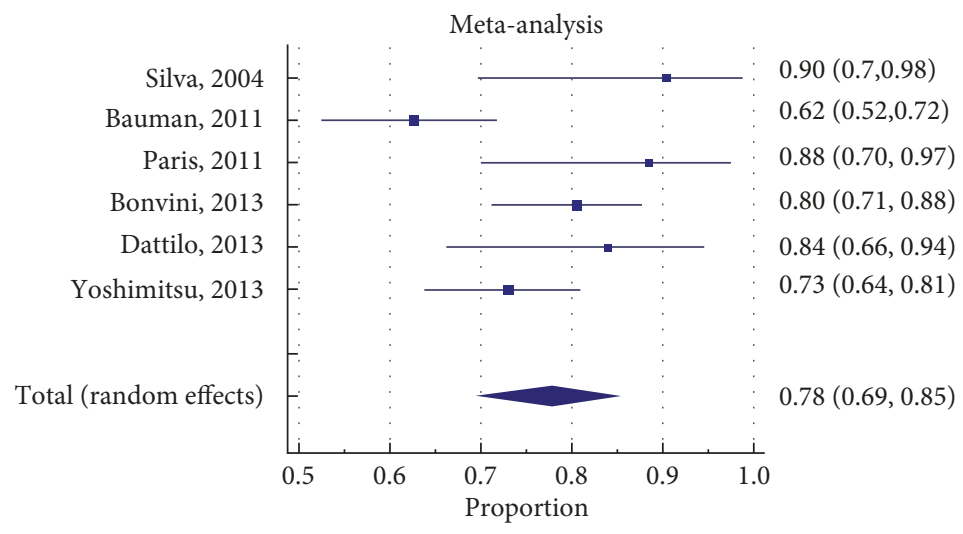

FIGURE 2: Forrest plot for PP (Primary patency) at 1 year in EVTSS (Endovascular therapy with selective stenting) group.

TABLE 3: Procedural details in different treatment groups.

\begin{tabular}{|c|c|c|c|}
\hline Variable & $\begin{array}{c}\text { EVT - Selective Stenting } \\
\mathrm{n}(\%)\end{array}$ & $\begin{array}{l}\text { EVT- Routine Stenting } \\
\mathrm{n}(\%) \\
\end{array}$ & $\begin{array}{l}\mathrm{CFE} \\
\mathrm{n}(\%)\end{array}$ \\
\hline Total stents & 123 & 330 & $552^{\infty}$ \\
\hline Balloon-Expandable stents & $15 / 110(13.6)^{\#}$ & $93(28.2)$ & DNA \\
\hline Self-Expandable stents & $95 / 110(86.4)^{\#}$ & $237(71.8)$ & DNA \\
\hline Bioabsorbable stents & $0(0)$ & $41(12.4)$ & DNA \\
\hline Covered stents & $0(0)$ & $17(5.1)$ & DNA \\
\hline Atherectomy & $72(10.6)$ & $15(4.9)$ & DNA \\
\hline Contralateral cross-over approach & $479(70.6)$ & $239(78.1)$ & DNA \\
\hline Ipsilateral retrograde approach & $104(15.3)$ & $38(12.4)$ & DNA \\
\hline Stent Fracture & $0(0)$ & $6(1.8)$ & DNA \\
\hline Stent Re puncture & $2(1.6)$ & $24(7.2)$ & DNA \\
\hline CFE only & DNA & DNA & $944(48.9)$ \\
\hline Hybrid CFE (CFE + endovascular procedure) & DNA & DNA & $794(41.1)$ \\
\hline CFE + Distal Revascularization & DNA & DNA & $401 / 1721(23.3)^{8}$ \\
\hline CFE + Proximal Revascularization & DNA & DNA & $495 / 1721(28.7)^{8}$ \\
\hline CFE + Proximal and Distal Revascularization & DNA & DNA & $81 / 1683(4.8)^{\pi}$ \\
\hline Patch angioplasty & DNA & DNA & $1325 / 1837(72.1)^{*}$ \\
\hline Profundaplasty & DNA & DNA & $414 / 743(55.7)^{\varphi}$ \\
\hline Concomitant Bypass & DNA & DNA & $110(5.6)$ \\
\hline
\end{tabular}

[\# 7 out of 8 studies $\mid \pi 11$ out of 13 studies | g 12 out of 13 studies $\mid * 12$ out of 13 studies $\mid \infty$ concomitant proximal or distal stents (not in CFA)] DNA-Does not apply.

at one year were $8 \%(95 \%$ CI $4-13 \%)$ for EVT-RS, $19 \%(95 \%$ CI 14-23\%) for EVT-SS, and 4.5\% (95\% CI 1-9\%) for CFE (supplementary file Figures 8-13). The CIs of CFE and EVT RS overlap while CI of EVT SS does not overlap with either EVT RS or CFE. Hence while superior to selective stenting, both routine stenting and endarterectomy had comparable rates of target lesion revascularization at 1 year. Table 4 summarizes the results elaborated above. Table 5 describes the studies that were included in the meta-analysis.

PP at maximum follow-up in EVT-RS was 83.7\% (95\% CI 74-91\%) (Figure 14 supplementary file), in CFE group was $88.3 \%$ (95\% CI 81-94\%) (Figure 15). Thus, the PP was comparable in the two groups. The maximum follow-up was similar in the EVT-RS and CFE groups but much lower in the EVT-SS group and thus not amenable for comparison. The average maximum follow in EVT-RS was 66.9 months (range 28-158), EVT-SS was 32.1 (range 16-36), and CFE was 80.01 (range 19-168).

3.7. Mortality and Complication Rates. The pooled mortality at 30 days was $0.8 \%$ (95\% CI 0.1-2\%) for EVT-RS, $1 \%$ (95\% CI $0.4-2 \%$ ) for EVT-SS, and 1.3\% (95\% CI 0.6-2\%) for CFE (supplementary file Figures 16-21). There was no statistically significant difference between the treatment strategies in this regard as CIs of the three groups overlap. The pooled rate of local complications for EVT-RS group was 5\% (95\% CI 2$10 \%)$ while the EVT-SS had 7\% local complication rates $(95 \%$ CI 3.3 to $11.8 \%$ ) and CFE had a pooled local complication rate of $22 \%$ (95\% CI 14-32\%) (supplementary file Figures 22-27). The pooled rate of amputations was 3\% (95\% CI 1-6\%) for 
TABLE 4: Clinical efficacy as demonstrated by pooled analysis of identified outcomes between the different treatment strategies.

\begin{tabular}{|c|c|c|c|}
\hline Variable & EVT - Selective Stenting & EVT- Routine Stenting & CFE \\
\hline $\begin{array}{l}\text { Primary Patency at } 1 \mathrm{yr} \\
{[95 \% \mathrm{CI}]\left[\mathrm{I}^{2}, \mathrm{p}\right]}\end{array}$ & $78 \%[69-85][69.37 \%, p=0.006]$ & $84 \%[75-92][75.52 \%, p=0.0004]$ & $93 \%[90-96][74.91 \%, \mathrm{p}<0.0001]$ \\
\hline $\begin{array}{l}\text { Primary Assisted Patency at } \\
1 \mathrm{yr}[95 \% \mathrm{CI}]\left[\mathrm{I}^{2}, \mathrm{p}\right]\end{array}$ & $86 \%$ [67-97] [91.8\%, p < 0.0001] & $94 \%$ [83-99] [81.29\%, $\mathrm{p}=0.0011]$ & $97 \%$ [94-99] [90.87\%, $\mathrm{p}<0.0001]$ \\
\hline $\begin{array}{l}\text { Target Lesion } \\
\text { revascularization at } 1 \mathrm{yr} \\
{[95 \% \mathrm{CI}]\left[\mathrm{I}^{2}, \mathrm{p}\right]} \\
\end{array}$ & $19 \%[14-23][22.3 \%, \mathrm{p}=0.27]$ & $8 \%[4-13][38.76 \%, p=0.1628]$ & $4.5 \%[1-9][85.07 \%, \mathrm{p}<0.0001]$ \\
\hline $\begin{array}{l}\text { Amputations }[95 \% \mathrm{CI}]\left[\mathrm{I}^{2} \text {, }\right. \\
\text { p] }\end{array}$ & $3 \%[1.3$ to 5.3$][14.5 \%, \mathrm{p}=0.31]$ & $3 \%[1-6][0.36 \%, p=0.4]$ & $4.5[2.5-6.8][75.32 \%, \mathrm{p}<0.0001]$ \\
\hline $\begin{array}{l}30 \text { days mortality }[95 \% \mathrm{CI}] \\
{\left[\mathrm{I}^{2}, \mathrm{p}\right]}\end{array}$ & $1 \%[0.4-2][0 \%, \mathrm{p}=0.43]$ & $0.8 \%[0.1-2][0 \%, \mathrm{p}=0.9]$ & $1.3 \%[0.6-2][0 \%, \mathrm{p}=0.56]$ \\
\hline $\begin{array}{l}\text { Local complications }[95 \% \\
\text { CI }]\left[\mathrm{I}^{2}, \mathrm{p}\right]\end{array}$ & $\%$ [3.3 to 11.8$][77.08 \%, p=0.0001]$ & $5.2[1.8-10.2][56.89 \%, \mathrm{p}=0.04]$ & $22.3 \%[13.8-32][95.2 \%, \mathrm{p}<0.0001]$ \\
\hline
\end{tabular}

An $\mathrm{I}^{2}$ more than $50 \%$ and a p less than 0.05 showing heterogeneity. Yr: year and CI: confidence interval.

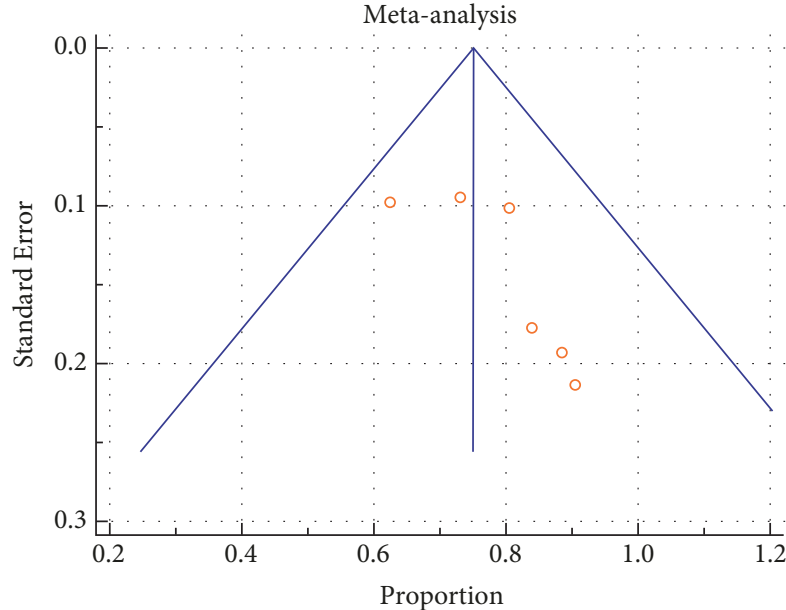

FIGURE 3: Funnel plot of studies regarding PP at 1 year in EVT SS by Egger test: asymmetrical.

EVT-RS, 4\% (95\% CI 2.4 to 6.0\%) for EVT-SS, and 4.5\% (95\% CI 2.5 to $6.8 \%$ ) for CFE (supplementary file Figures 28-33) (Tables 4 and 6).

Mortality at maximum follow-up in CFE group was 23.1\% (95\% CI 14-33\%) and EVT-RS was 5.3\% (95\% CI 1.6$11 \%$ ) (Figures 34-36, supplementary file). Thus, mortality at maximum follow-up is much smaller (statistically significant) in EVT-RS than CFE. As mentioned above, the maximum follow-up was similar in the EVT-RS and CFE groups but much lower in the EVT-SS group.

3.8. Heterogeneity. Heterogeneity between the included studies using $\mathrm{I}^{2}$ was documented for PP statistics at 1 year in all groups (EVTSS 69.4\%, p=0.006, EVT RS 75.5\%, p=0.0004, CFE $74.9 \%$, and $p<0.0001)$. Heterogeneity in studies was not significant for TLR at one year in EVT SS $(22.3 \%, \mathrm{p}=$ 0.27 ) and EVTRS $(38.7 \%, \mathrm{p}=0.16)$, while it was significant in CFE (85.1\%, $\mathrm{p}<0.0001)$. Heterogeneity in studies was not significant for data on amputations in EVT SS $(14.5 \%, \mathrm{p}=0.31)$ and EVTRS $(0.36 \%, \mathrm{p}=0.4)$, while it was significant in CFE $(75.3 \%, \mathrm{p}<0.0001)$. Heterogeneity in studies was not seen for 30 days of mortality in any group (EVTSS $0 \%, \mathrm{p}=0.43$, EVTRS $0 \%$, and $\mathrm{p}=0.9$; CFE $0 \%, \mathrm{p}=0.56)$. Heterogeneity between the included studies using $\mathrm{I}^{2}$ was documented in local complications in all groups (EVTSS 77.1\%, $\mathrm{p}=0.0001$, EVTRS 56.9\%, $\mathrm{p}=0.04$, CFE 95.2\%, and $\mathrm{p}<0.0001$ ) (Table 1). Funnel plots were used to assess for publication bias. Asymmetric funnel plots were seen in EVTSS group for PP at 1 year (Figure 3) and TLR at one year (Figure 9, supplementary file) showing possible publication bias. Asymmetric funnel plots were not seen in EVTRS group. Asymmetric funnel plots were seen in CFE group for PP at one year (Figure 7), amputations (Figure 33, supplementary file), and local complications (Figure 27, supplementary file) possibly due to publication bias.

\section{Discussion}

In our analysis, one-year PP in EVT-RS and CFE was similar (EVT-RS 84\%, 95\% CI 75- 92\%; CFE 93\%, and 95\% CI 90$96 \%)$. The rate of local complications was higher in CFE (22\%, 95\% CI 14- 32\%) compared to either EVT-RS (5\%, 95\% CI 2$10 \%$ ) or EVT-SS (7\%, 95\% CI 3-11\%). Hence, endovascular therapy with routine stenting had similar PP at one year as compared to CFE but had lesser complications. However, both CFE and EVT-RS had better one-year PP than EVT-SS $78 \%$ (95\% CI 69-85\%). Thus, selective stenting of CFA is not a good treatment strategy. At maximum follow-up CFE and EVT-RS continued to have similar PP. However, the mortality in the CFE group was significantly higher than in the EVT-RS group.

Our results are similar to the results of TECCO trial [(Traitement des Lésions Athéromateuses de l'Artère Fémorale Commune par Technique Endovasculaire Versus Chirurgie Ouverte (Endovascular Versus Open Repair of the Common Femoral Artery))] which compared EVT-RS with CFE. TECCO trial showed that there was no significant difference in primary patency (one-year PP in CFE90\%, EVT-90\%), sustained clinical improvement, and target 


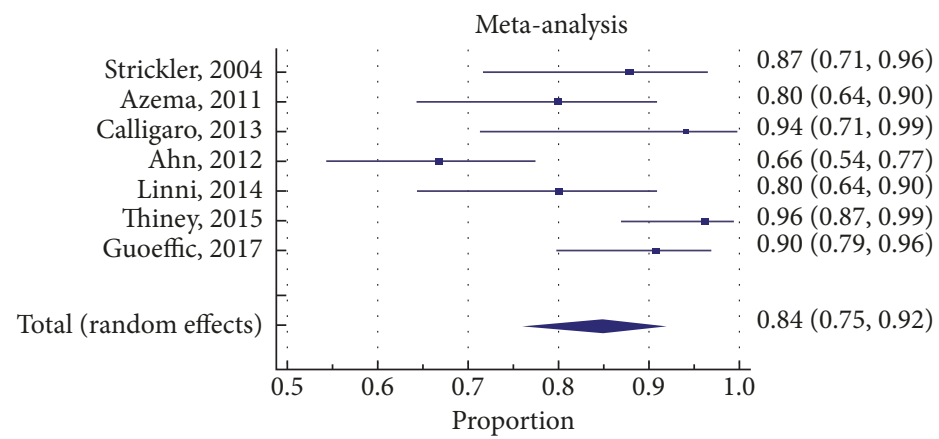

FIGURE 4: Forest plot for primary patency at 1 year in EVT RS group.

TABLE 5: Showing the details of the studies included in the meta-analysis.

\begin{tabular}{|c|c|c|c|c|c|c|}
\hline Study & YOP & Country & Design & Study Period & Total Duration in Years & Mean age \\
\hline Stickler [9] & 2004 & Switzerland & Retrospective Analysis & $1995-2002$ & 7 & 70 \\
\hline Azema/Nasr $[12,13]$ & 2011 & France & prospective cohort & $2006-2008$ & 3 & 67.3 \\
\hline Calligaro [14] & 2011 & USA & Retrospective Analysis & $2005-2010$ & 6 & 69.5 \\
\hline Ahn [15] & 2012 & USA & Retrospective Analysis & 2009-2011 & 3 & 67.2 \\
\hline Linni: Stent [10] & 2014 & Austria & RCT & $2011-2013$ & 2 & 71.6 \\
\hline Thiney [16] & 2015 & France & Retrospective Analysis & 2009-2013 & 4 & 68 \\
\hline Guoeffic: Stent [11] & 2017 & France & Prospective cohort & $2011-2013$ & 2.5 & 68 \\
\hline \multicolumn{7}{|c|}{ EVT Selective Stenting group } \\
\hline Silva [17] & 2004 & USA & $\begin{array}{c}\text { retrospective review of } \\
\text { prospectively managed } \\
\text { database } \\
\end{array}$ & na & na & 64.9 \\
\hline Bauman [18] & 2011 & switzerland & $\begin{array}{c}\text { retrospective review of } \\
\text { prospectively managed } \\
\text { database }\end{array}$ & 1995-2009 & 15 & 72 \\
\hline Paris [19] & 2011 & USA & Retrospective Analysis & 1994-2009 & $15 \mathrm{y}$ & 68.9 \\
\hline Bonvini [20] & 2013 & Germany & Retrospective Analysis & 1996-2007 & 11 & 68.7 \\
\hline Dattilo [21] & 2013 & USA & Retrospective Analysis & 2006-2011 & 6 & 62.8 \\
\hline Davies [22] & 2013 & $\mathrm{UK}$ & Retrospective Analysis & 2006-2012 & $6 y$ & 71 \\
\hline Yoshimitsu [23] & 2013 & Japan & Retrospective Analysis & $2001-2010$ & 10 & 71 \\
\hline Mehta [41] & 2016 & USA & $\begin{array}{c}\text { prospectively maintained } \\
\text { multicenter database }\end{array}$ & $2006-2013$ & 7 & 68 \\
\hline \multicolumn{7}{|c|}{ CFE group } \\
\hline Nelson [24] & 2002 & USA & Retrospective Analysis & $1997-2000$ & 3 & 70.8 \\
\hline Kang [25] & 2008 & USA & $\begin{array}{c}\text { retrospective review of } \\
\text { prospectively gathered data }\end{array}$ & $2002-2005$ & 4 & 74 \\
\hline Chang [26] & 2008 & USA & Retrospective review & $1997-2006$ & 10 & 73.5 \\
\hline Kechegias [27] & 2008 & Finland & Retrospective Review & $1983-2006$ & 13 & 72.4 \\
\hline Ballotta [28] & 2010 & Italy & prospective cohort & $2000-2007$ & 8 & 68 \\
\hline Desai [29] & 2011 & $\mathrm{UK}$ & retrospective review & 1996-2008 & 13 & 71.2 \\
\hline Malgor [30] & 2012 & USA & Retrospective Analysis & $1997-2008$ & 11 & 68 \\
\hline Dufranc [31] & 2015 & France & prospective cohort & $2010-2012$ & 3 & 68.7 \\
\hline Nishibe [32] & 2015 & japan & retrospective review & $2010-2014$ & 4 & 67 \\
\hline Linni [10] & 2014 & Austria & RCT & $2011-2013$ & 2 & 67.4 \\
\hline Wieker [33] & 2016 & Germany & retrospective 2 center & $2006-2012$ & 6 & 69.4 \\
\hline Kuma [34] & 2016 & japan & retrospective multicenter & 1998-2014 & 17 & 68.6 \\
\hline Guoeffic [11] & 2017 & France & RCT & $2011-2013$ & 2.5 & 69.4 \\
\hline
\end{tabular}


TABLE 6: Details of complications.

Complications reported in EVT Routine Stenting

\begin{tabular}{lr} 
& \multicolumn{1}{c}{$\mathrm{n}[\%]$} \\
\hline Hematoma & $2(0.65)$ \\
\hline Inflow/outflow vessel stenosis & $2(0.65)$ \\
\hline Stent occlusion & $2(0.65)$ \\
\hline Thrombosis & $1(0.32)$ \\
\hline Vascular perforation & $1(0.32)$ \\
\hline Local infection & $1(0.32)$ \\
\hline Stroke & $1(0.32)$ \\
\hline In stent restenosis & $32(10.4)$ \\
\hline Strut failure & $1(0.32)$ \\
\hline Stent fracture & $6(1.9)$ \\
\hline
\end{tabular}

\begin{tabular}{|c|c|}
\hline \multicolumn{2}{|c|}{$\begin{array}{l}\text { Complications reported in EVT Selective Stenting } \\
\qquad[n=678 \text { limbs }]\end{array}$} \\
\hline Thromboembolic complications & $8(1.2)$ \\
\hline Pseudo aneurysm & $2(0.29)$ \\
\hline AV Fistula & $1(0.14)$ \\
\hline Perforation/CFA dissection & $3(0.44)$ \\
\hline Pulmonary edema & $1(0.14)$ \\
\hline Myocardial Infarction & $3(0.44)$ \\
\hline Retroperitoneal bleed & $1(0.14)$ \\
\hline Groin hematomas & $22(3.2)$ \\
\hline Acute Kidney Injury & $1(0.14)$ \\
\hline Sepsis & $1(0.14)$ \\
\hline
\end{tabular}

Complications reported in CFE

[n=1930 limbs]

\begin{tabular}{lr}
\hline Wound/local infection & $142(7.3)$ \\
\hline Myocardial infarction & $21(1.1)$ \\
\hline Proximal external iliac artery dissection & $1(0.05)$ \\
\hline Cardiac complications & $69(3.5)$ \\
\hline Pulmonary complications & $21(1.1)$ \\
\hline Thrombotic and embolic complications & $9(0.46)$ \\
\hline Inguinal lymph leaks & $49(2.5)$ \\
\hline Hematoma/bleeding comp & $30(1.5)$ \\
\hline Seroma & $6(0.31)$ \\
\hline Acute Kidney Injury & $24(1.2)$ \\
\hline Neurological complications & $10(0.5)$ \\
\hline Delayed wound healing & $19(0.98) \_$ \\
\hline Sepsis & $4(0.21)$ \\
\hline
\end{tabular}

revascularization rates between $\mathrm{CFE}$ and EVT-RS groups. The rate of perioperative complications (26\% in CFE vs. $12.5 \%$ in EVT) and hospital stay $(6.3 \pm 3$ in CFE vs.3.2 \pm 2.9 in EVT) was more in the CFE group compared to EVT-RS group. Self-expanding nitinol stents were used in this study [11]. The only other RCT comparing EVT-RS and CFE was published by Linni et al. in 2014 [10]. While the patency rates were significantly lower in the stented group compared to CFE (one-year PP was $80 \%$ in BAS vs. $100 \%$ in CFE; $\mathrm{p}=0.007$ ), the stents used in this study were bioabsorbable Poly L Lactic Acid stents that have struggled to demonstrate better outcomes in the coronary vasculature as well $[22,39-$ 41]. The worse performance of BAS may be related to the lower radial strength of bioresorbable scaffolds and hence poor patency rates. The bioresorbable stent technology has shown inferiority in the coronary arteries in the Absorb III and AIDA trial where they failed to deliver better long-term outcomes in comparison to drug-eluting nitinol stents and were associated with increased stent thrombosis, including late stent thrombosis [22, 39-41].

Stent fractures, which are cited as a major problem with endovascular therapy of CFA, were reported in only $1.9 \%$ of 


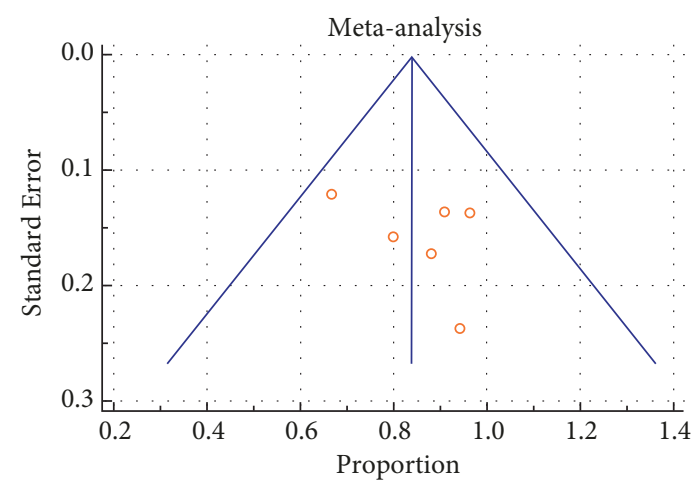

FIGURE 5: Funnel plot of studies regarding PP at I year in EVTRS group by Egger test. Symmetrical.

the patient included in the routine stenting group. Twentyfour stents were repunctured for vascular access in the routine stenting group; no complications were reported. This shows that stent fracture in CFA location is uncommon and concerns regarding later access may not be a reason to exclude endovascular therapy as a possible procedure of choice in suitable candidates. In general, stent fractures are a result of stent design, the anatomy of the stented segment, biomechanical forces, and plaque morphology [42]. The design of stents used for CFA atherosclerotic disease has evolved. Majority of the stents deployed in the endovascular therapy groups were self-expanding. Percentage of self-expanding vs. balloon-expanding stents was $71.8 \%$ vs $28.2 \%$ in EVTRS group whereas it was $86.4 \%$ vs $13.6 \%$ in EVT-SS. Selfexpanding stents are largely replacing balloon expanding stents in lower limb arterial disease. Most self-expanding stents are now made of nitinol which is an alloy of nickel and titanium. An important feature of nitinol that is beneficial in the self-expanding design of stents is its thermal shape memory and super-elasticity $[10,36,42-44]$. On the contrary, stiffer balloon-expandable stents have less radial strength and high chances of deformation and fracture [45].

As described previously CFE had more complications than EVT-RS or EVT-SS. The most common complications reported in EVT-RS group were in-stent restenosis (10.4\%), stent fracture $(1.9 \%)$, hematoma $(0.65 \%)$, inflow/outflow vessel stenosis $(0.65 \%)$, stent occlusion $(0.65 \%)$, and thrombosis $(0.32 \%)$ [Table 6 ]. The most common complications in EVT-SS group were hematomas (3.2\%), thromboembolic complications (1.17\%), and perforation/dissection (0.44\%). While local wound infections are practically non-existent in the EVT groups, it was the most common complication encountered in CFE (7.3\%) followed by cardiac complications (including myocardial infarction) (4.58\%), inguinal lymph leaks $(2.5 \%)$, hematoma/bleeding (1.55\%), and acute renal failure (1.24\%). Systemic complications were more common in the CFE group. The mean age of patients was 68.4 years in EVT-SS group, 68.8 years in EVT-RS group, and 69.9 years in CFE group. Majority of patients were men in our analysis (61.7\% in EVT-SS, 68.8\% EVT-RS, and 69.9\% in CFE; Table 1) and based on prior epidemiology studies peripheral arterial disease is more common in men as compared to women $[41,46]$.

\section{Limitations}

Only two RCTs were available for comparison. One used bioabsorbable stents which are not effective stents. Ideally, a meta-analysis of RCTs would be best but due to nonavailability of RCTs on the current topic, a proportional metaanalysis provides the best evidence. There was significant heterogeneity in EVTSS group for PP, primary assisted patency, and local complications. The funnel plots for PP and TLR are asymmetric possibly due to publication bias. Heterogeneity was also seen for PP, primary assisted patency and local complications in EVTRS group. The funnel plots in EVT RS group were symmetric showing less likelihood of publication bias. Heterogeneity was seen in CFE studies for PP, primary assisted patency, TLR, amputations, and local complications. Funnel plots are asymmetrical for primary patency, primary assisted patency, amputations, and local complications from possible publication bias. Overall, the heterogeneity was possibly due to lack of consistency in the design of studies, patient selection and reporting of complications. The higher percentage of CLI patients in CFE group (58.1\%) than in EVT-selective stenting (35.1\%) or EVTroutine stenting $(33.5 \%)$ could have affected the outcomes studied in this study. A study matched for CLI is lacking currently in literature and if done may give more accurate outcomes.

\section{Conclusion}

Endovascular therapy with routine stenting strategy has comparable primary patency and target lesion revascularization rates as compared to common femoral endarterectomy. Complications and mortality of CFE are higher than EVT. These findings support endovascular therapy as an alternative in suitable candidates. There is a need for large randomized controlled trials to compare outcomes of routine stenting strategy to common femoral endarterectomy.

\section{Data Availability}

The data used to support the findings of this study are available from the corresponding author upon request.

\section{Disclosure}

Part of this work was presented at the American College of Cardiology 2019 Scientific sessions, New Orleans, as an abstract, published in 'Journal of the American College of Cardiology Volume 73, Issue 9, Supplement 1, 12 March 2019, Page 2065' as an abstract with the title 'Clinical Outcome of Endovascular Therapy with Routine Stenting Versus Endarterectomy For Common Femoral Artery Atherosclerotic Disease: A Proportional Meta-Analysis'. 


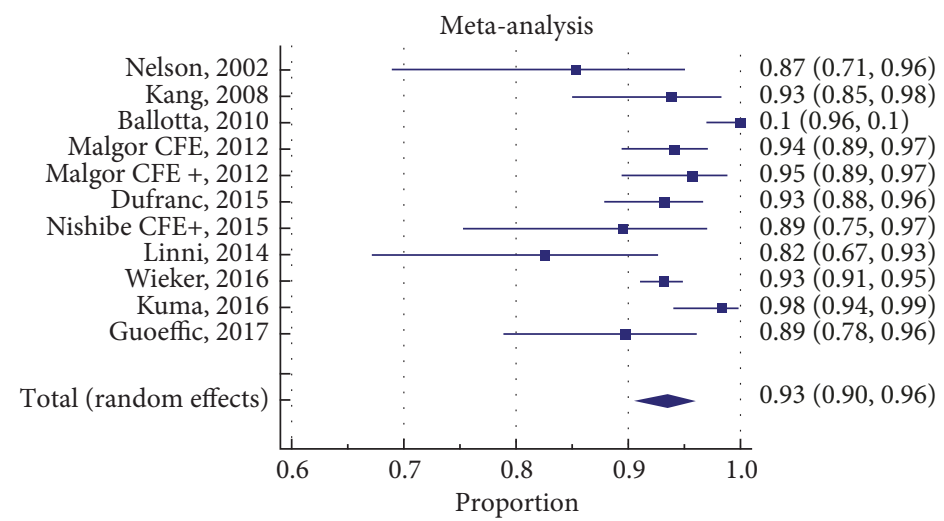

Figure 6: Forrest plot for PP at 1 year in CFE group.

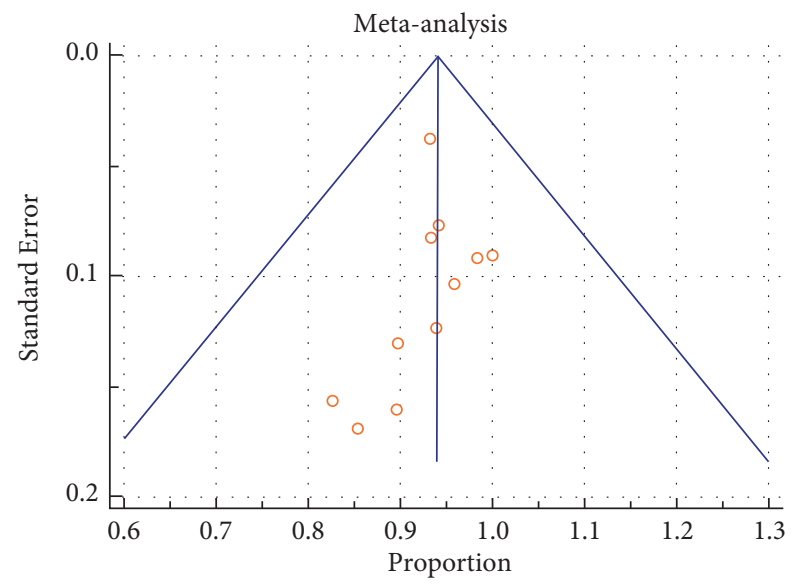

FIGURE 7: Funnel plot of studies about PP at I year in CFE group by Egger test: asymmetrical.

\section{Conflicts of Interest}

The authors report no financial relationships or conflicts of interest regarding the content herein.

\section{Supplementary Materials}

Figure 8: Forrest plot for TLR 1 year in EVT SS group. Figure 9: Funnel plot for TLR 1 year EVT SS group: asymmetrical. Figure 10: Forrest plot for TLR at 1 year in EVT RS group. Figure 11: Funnel plot for TLR at 1 year in EVT RS group: symmetrical. Figure 12: Forrest plot for TLR at 1 year in CFE group. Figure 13: Funnel plot for TLR at 1 year in CFE group: symmetrical. Figure 14: Forrest plot for PP at maximum follow-up in EVT-RS: PP- 83.7\% (95\% CI 0.740.91). Figure 15: Forrest plot for PP in CFE at maximum follow-up: PP- 88.34\% (95\% CI 0.81-0.94). Figure 16: Forrest plot for mortality at 30 days in EVT SS group. Figure 17: Funnel plot for mortality 30 days in EVT SS group: symmetrical. Figure 18: Forrest plot for mortality 30 days in EVT RS group. Figure 19: Funnel plot for mortality at 30 days in EVT RS group: symmetrical. Figure 20: Forrest plot for mortality at 30 days in CFE group. Figure 21: Funnel plot for mortality at 30 days in CFE group: symmetrical. Figure 22: Forrest plot for local complications in EVTSS group. Figure 23: Funnel plot for local complications in EVT SS group: symmetrical. Figure 24: Forrest plot for local complications in EVT RS group. Figure 25: Funnel plot for local complications, EVTRS group: symmetrical. Figure 26: Forrest plot for local complications in CFE group. Figure 27: Funnel plot for Local complications in CFE group: asymmetrical. Figure 28: Forrest plot for amputations in EVT SS group. Figure 29: Funnel plot for amputations in EVT SS group: symmetrical. Figure 30: Forrest plot for amputations in EVT RS group. Figure 31: Funnel plot for amputations in EVT SS group: symmetrical. Figure 32: Forrest plot for amputations in CFE group. Figure 33: Funnel plot for amputations in CFE group: asymmetrical. Figure 34: Forrest plot for mortality at max follow-up in CFE group. Mortality was 23.1\% (95\% CI 0.140.33 ). Figure 35: Forrest plot for mortality at max followup in EVT-RS group. Mortality was 5.33\% (95\% CI 0.0160.11). Figure 36: Forrest plot for mortality at max follow-up in EVT-SS group. Mortality was 10.9\% (95\% CI 0.03-0.22). (Supplementary Materials)

\section{References}

[1] K. W. Johnston, M. Rae, S. A. Hogg-Johnston et al., "5-year results of a prospective study of percutaneous transluminal angioplasty," Annals of Surgery, vol. 206, no. 4, pp. 403-413, 1987.

[2] I. Töpel, M. Wiesner, C. Uhl, T. Betz, and M. G. Steinbauer, "Retrograde thrombendarterectomy vs. ilio-femoral bypass in unilateral iliac TASC C and D lesions," Vasa - European Journal of Vascular Medicine, vol. 44, no. 3, pp. 211-219, 2015.

[3] K. W. Johnston, M. Rae, S. A. Hogg-Johnston et al., "5-year results of a prospective study of percutaneous transluminal angioplasty," Annals of Surgery, vol. 206, no. 4, pp. 403-413, 1987.

[4] M. Nasr, R. McCarthy, J. Hardman, A. Chalmers, and M. Horrocks, "The increasing role of percutaneous transluminal angioplasty in the primary management of critical limb ischaemia," European Journal of Vascular and Endovascular Surgery, vol. 23, no. 5, pp. 398-403, 2002.

[5] K. J. Molloy, A. Nasim, N. J. M. London et al., "Percutaneous transluminal angioplasty in the treatment of critical limb ischemia," Journal of Endovascular Therapy, vol. 10, no. 2, pp. 298-303, 2003. 
[6] S. M. Surowiec, M. G. Davies, S. W. Eberly et al., "Percutaneous angioplasty and stenting of the superficial femoral artery," Journal of Vascular Surgery, vol. 41, no. 2, pp. 269-278, 2005.

[7] J. H. Black III, G. M. LaMuraglia, C. J. Kwolek, D. C. Brewster, M. T. Watkins, and R. P. Cambria, "Contemporary results of angioplasty-based infrainguinal percutaneous interventions," Journal of Vascular Surgery, vol. 42, no. 5, pp. 932-939, 2005.

[8] M. F. Conrad, R. P. Cambria, D. H. Stone et al., "Intermediate results of percutaneous endovascular therapy of femoropopliteal occlusive disease: A contemporary series," Journal of Vascular Surgery, vol. 44, no. 4, pp. 762-769, 2006.

[9] H. Stricker and V. Jacomella, "Stent-assisted angioplasty at the level of the common femoral artery bifurcation: Midterm outcomes," Journal of Endovascular Therapy, vol. 11, no. 3, pp. 281-286, 2004.

[10] K. Linni, A. Ugurluoglu, W. Hitzl, M. Aspalter, and T. Hölzenbein, "Bioabsorbable Stent Implantation vs. Common Femoral Artery Endarterectomy: Early Results of a Randomized Trial," Journal of Endovascular Therapy, vol. 21, no. 4, pp. 493-502, 2014.

[11] Y. Gouëffic, N. Della Schiava, F. Thaveau et al., "Stenting or surgery for de novo common femoral artery stenosis," JACC: Cardiovascular Interventions, vol. 10, no. 13, pp. 1344-1354, 2017.

[12] L. Azéma, J. Davaine, B. Guyomarch et al., "Endovascular repair of common femoral artery and concomitant arterial lesions," European Journal of Vascular and Endovascular Surgery, vol. 41, no. 6, pp. 787-793, 2011.

[13] B. Nasr, A. Kaladji, P.-A. Vent et al., "Long-term outcomes of common femoral artery stenting," Annals of Vascular Surgery, vol. 40, pp. 10-18, 2017.

[14] K. D. Calligaro, P. Balraj, N. Moudgill, A. Rao, M. J. Dougherty, and J. Eisenberg, "Results of polytetrafluoroethylene-covered nitinol stents crossing the inguinal ligament," Journal of Vascular Surgery, vol. 57, no. 2, pp. 421-426, 2013.

[15] S. S. Ahn, M. J. Uceda, K. R. Kollmeyer, C. A. Ferrara, and P. V. Uceda, "Outpatient common femoral artery stenting: midterm outcomes," Journal of Vascular Surgery, vol. 56, no. 2, p. 582, 2012.

[16] P.-O. Thiney, A. Millon, T. Boudjelit, N. Della Schiava, P. Feugier, and P. Lermusiaux, "Angioplasty of the Common Femoral Artery and Its Bifurcation," Annals of Vascular Surgery, vol. 29, no. 5, pp. 960-967, 2015.

[17] J. A. Silva, C. J. White, H. Quintana, T. J. Collins, J. S. Jenkins, and S. R. Ramee, "Percutaneous revascularization of the common femoral artery for limb ischemia," Catheterization and Cardiovascular Interventions, vol. 62, no. 2, pp. 230-233, 2004.

[18] F. Baumann, M. Ruch, T. Willenberg et al., "Endovascular treatment of common femoral artery obstructions," Journal of Vascular Surgery, vol. 53, no. 4, pp. 1000-1006, 2011.

[19] C. L. Paris, C. J. White, T. J. Collins et al., "Catheter-based therapy of common femoral artery atherosclerotic disease," Vascular Medicine, vol. 16, no. 2, pp. 109-112, 2011.

[20] R. F. Bonvini, A. Rastan, S. Sixt et al., "Endovascular treatment of common femoral artery disease," Journal of the American College of Cardiology, vol. 58, no. 8, pp. 792-798, 2011.

[21] P. B. Dattilo, T. T. Tsai, R. Kevin Rogers, and I. P. Casserly, "Acute and medium-term outcomes of endovascular therapy of obstructive disease of diverse etiology of the common femoral artery," Catheterization and Cardiovascular Interventions, vol. 81, no. 6, pp. 1013-1022, 2013.
[22] R. S. Davies, W. Adair, A. Bolia, G. Fishwick, R. D. Sayers, and M. J. McCarthy, "Endovascular treatment of the common femoral artery for limb ischemia," Vascular and Endovascular Surgery, vol. 47, no. 8, pp. 639-644, 2013.

[23] Y. Soga, Y. Tomoi, K. Sato et al., "Clinical outcome after endovascular treatment for isolated common femoral and popliteal artery disease," Cardiovascular Intervention and Therapeutics, vol. 28, no. 3, pp. 250-257, 2013.

[24] P. R. Nelson, R. J. Powell, M. L. Schermerhorn et al., "Early results of external iliac artery stenting combined with common femoral artery endarterectomy," Journal of Vascular Surgery, vol. 35, no. 6, pp. 1107-1113, 2002.

[25] J. L. Kang, V. I. Patel, M. F. Conrad, G. M. LaMuraglia, T. K. Chung, and R. P. Cambria, "Common femoral artery occlusive disease: contemporary results following surgical endarterectomy," Journal of Vascular Surgery, vol. 48, no. 4, pp. 872-877, 2008.

[26] R. W. Chang, P. P. Goodney, J. H. Baek, B. W. Nolan, E. M. Rzucidlo, and R. J. Powell, "Long-term results of combined common femoral endarterectomy and iliac stenting/stent grafting for occlusive disease," Journal of Vascular Surgery, vol. 48, no. 2, pp. 362-367, 2008.

[27] A. Kechagias, K. Ylönen, and F. Biancari, "Long-term outcome after isolated endarterectomy of the femoral bifurcation," World Journal of Surgery, vol. 32, no. 1, pp. 51-54, 2008.

[28] E. Ballotta, M. Gruppo, F. Mazzalai, and G. Da Giau, "Common femoral artery endarterectomy for occlusive disease: an 8-year single-center prospective study," Surgery, vol. 147, no. 2, pp. 268 274, 2010.

[29] M. Desai, J. Tsui, M. Davis et al., "Isolated endarterectomy of femoral bifurcation in critical limb ischemia: is restoration of inline flow essential?” Angiology, vol. 62, no. 2, pp. 119-125, 2011.

[30] R. D. Malgor, J. J. Ricotta II, T. C. Bower et al., "Common femoral artery endarterectomy for lower-extremity ischemia: Evaluating the need for additional distal limb revascularization," Annals of Vascular Surgery, vol. 26, no. 7, pp. 946-956, 2012.

[31] J. Dufranc, L. Palcau, M. Heyndrickx et al., "Technique and results of femoral bifurcation endarterectomy by eversion," Journal of Vascular Surgery, vol. 61, no. 3, pp. 728-733, 2015.

[32] T. Nishibe, K. Maruno, A. Iwahori et al., "The role of common femoral artery endarterectomy in the endovascular era," Annals of Vascular Surgery, vol. 29, no. 8, pp. 1501-1507, 2015.

[33] C. M. Wieker, E. Schönefeld, N. Osada et al., "Results of common femoral artery thromboendarterectomy evaluation of a traditional surgical management in the endovascular era," Journal of Vascular Surgery, vol. 64, no. 4, pp. 995-1001, 2016.

[34] S. Kuma, K. Tanaka, T. Ohmine et al., "Clinical outcome of surgical endarterectomy for common femoral artery occlusive disease," Circulation Journal, vol. 80, no. 4, pp. 964-969, 2016.

[35] J. P. Higgins, S. G. Thompson, J. J. Deeks, and D. G. Altman, "Measuring inconsistency in meta-analysis," The BMJ, vol. 327, pp. 557-560, 2003.

[36] R. Fontaine, M. Kim, and R. Kieny, "Surgical treatment of peripheral circulation disorders," Helvetica Chimica Acta, vol. 21, pp. 499-533, 1954.

[37] R. B. Rutherford, D. P. Flanigan, S. K. Gupta et al., "Suggested standards for reports dealing with lower extremity ischemia," Journal of Vascular Surgery, vol. 4, no. 1, pp. 80-94, 1986.

[38] R. F. Bonvini, A. Rastan, S. Sixt et al., "Endovascular treatment of common femoral artery disease: Medium-term outcomes of 
360 consecutive procedures," Journal of the American College of Cardiology, vol. 58, no. 8, pp. 792-798, 2011.

[39] S. G. Ellis, D. J. Kereiakes, D. C. Metzger et al., "Everolimuseluting bioresorbable scaffolds for coronary artery disease," The New England Journal of Medicine, vol. 373, no. 20, pp. 1905-1915, 2015.

[40] J. J. Wykrzykowska, R. P. Kraak, S. H. Hofma et al., "Bioresorbable scaffolds versus metallic stents in routine PCI," The New England Journal of Medicine, vol. 376, no. 24, pp. 2319-2328, 2017.

[41] M. Mehta, Y. Zhou, P. S. K. Paty et al., "Percutaneous common femoral artery interventions using angioplasty, atherectomy, and stenting," Journal of Vascular Surgery, vol. 64, no. 2, pp. 369$379,2016$.

[42] S. Adlakha, M. Sheikh, J. Wu et al., "Stent fracture in the coronary and peripheral arteries," Journal of Interventional Cardiology, vol. 23, no. 4, pp. 411-419, 2010.

[43] T. W. Duerig, A. R. Pelton, and D. Stöckel, “The utility of superelasticity in medicine," Bio-Medical Materials and Engineering, vol. 6, no. 4, pp. 255-266, 1996.

[44] J. Chen, T. Duerig, A. Pelton, and D. Stöckel, "An apparatus to measure the shape memory properties of nitinol tubes for medical applications," Journal de Physique IV, vol. 5, pp. 12471252, 1995.

[45] S. H. Duda, J. Wiskirchen, G. Tepe et al., "Physical properties of endovascular stents: An experimental comparison," Journal of Vascular and Interventional Radiology, vol. 11, no. 5, pp. 645654, 2000.

[46] M. H. Criqui and V. Aboyans, "Epidemiology of peripheral artery disease," Circulation Research, vol. 116, no. 9, pp. 15091526, 2015. 


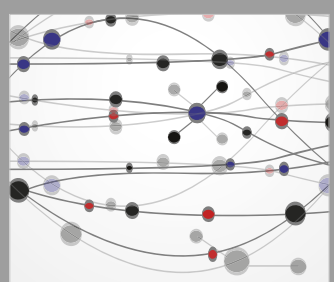

The Scientific World Journal
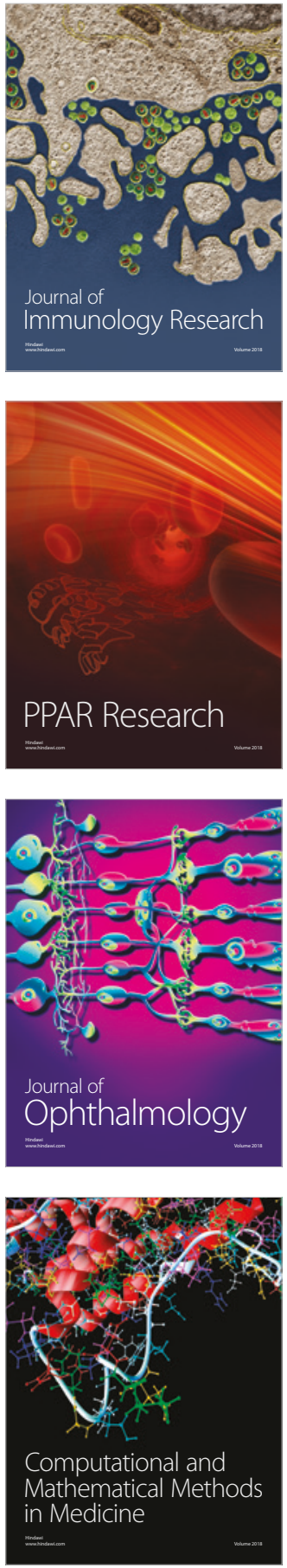

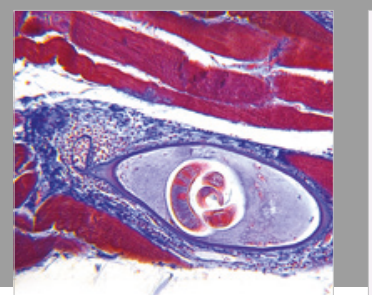

Gastroenterology Research and Practice

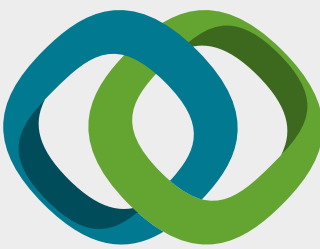

\section{Hindawi}

Submit your manuscripts at

www.hindawi.com
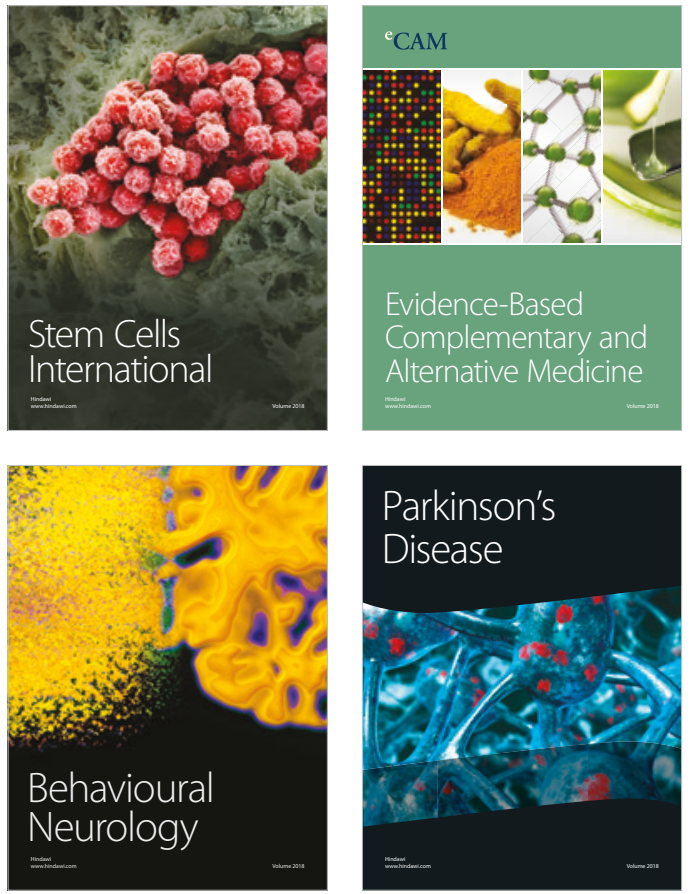

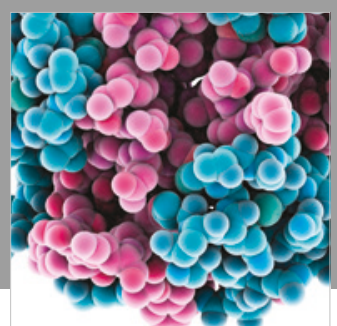

ournal of

Diabetes Research

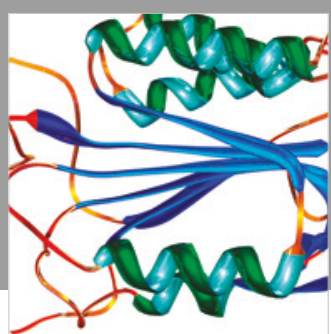

Disease Markers
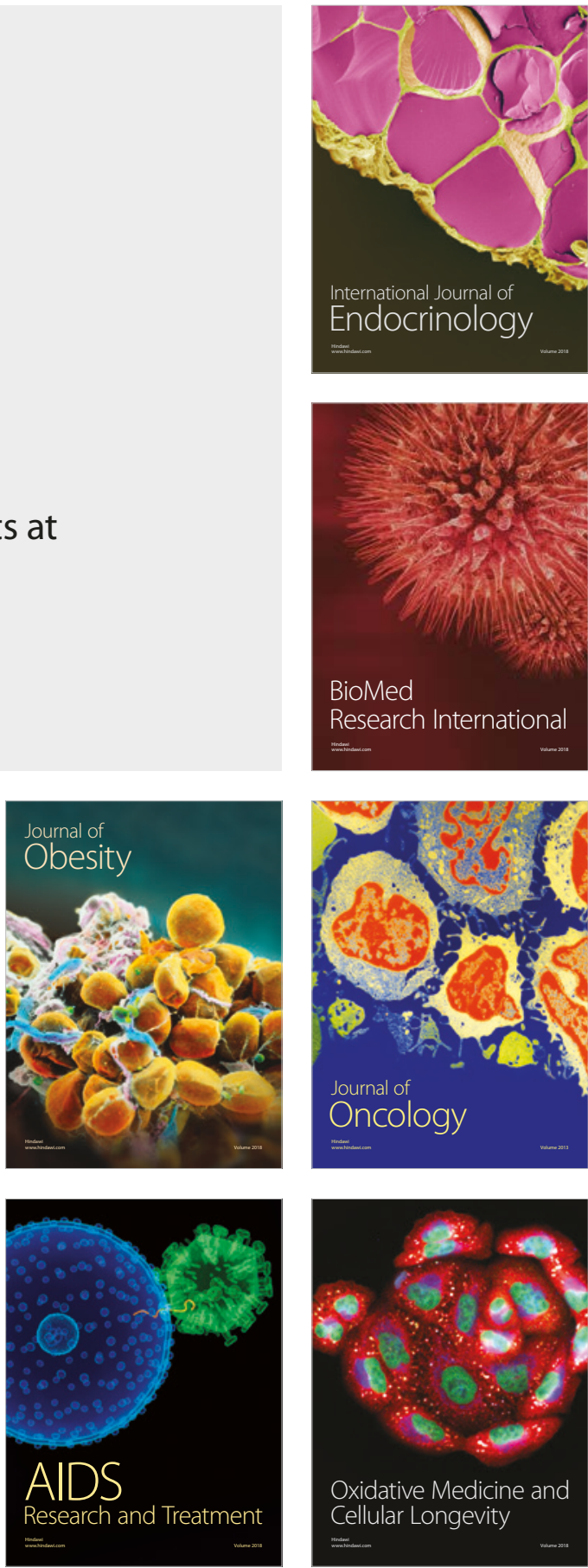